\title{
Performance and Quench Characteristics of a Pancake Coil Wound With the 2G YBCO Roebel Cable
}

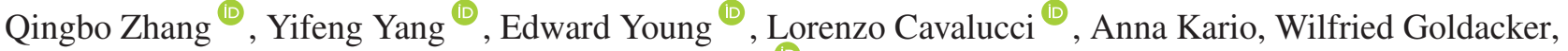 \\ Alexander Usoskin ${ }^{(1)}$, and Luca Bottura
}

\begin{abstract}
The YBCO Roebel cable is a promising option for the high-energy accelerator magnets and tokamak devices with high-current carrying capability and compactness. Referring to the complex current sharing and normal zone propagation, it is difficult to apply the traditional superconductor quench measurement methodology to such a long Roebel cable $(2 \mathrm{~m})$ in an adiabatic condition. An alternative method for the quench measurement is to make a Roebel pancake coil cowound with ribbon fiberglass. This paper starts reporting the methodology to wind the coil with impregnation process and the wire installment for the quench measurement. Some initial performance measurements and a first trial of the quench measurement were processed in LN2 at $77 \mathrm{~K}$ to show the robust performance of this Roebel cable at all the current contacts while maintaining the cable effectively adiabatic or similar to real magnets.
\end{abstract}

Index Terms-Roebel cable, pancake coil, high temperature superconductors, quench measurement.

\section{INTRODUCTION}

$\mathbf{T}$ HE high current carrying capability and strong pinning in high magnetic field make the $2 \mathrm{G}$ HTS Roebel cable desirable for future high energy accelerators at CERN and tokamak devices [1]-[4]. Some research has already shown a more complex current sharing and normal zone propagation is expected for Roebel cables than those found in traditional superconducting composites due to the non-continuous touch contacts among the Roebel strands [1]-[3], [5]-[8]. Therefore, the standard method of experimental quench studies on adiabatic short conductors

Manuscript received September 28, 2017; accepted January 24, 2018. Date of publication January 26, 2018; date of current version February 16, 2018. This work was supported in part by the European Commission under FP7-EuCARD2 Grant Agreement 312453. (Corresponding author: Qingbo Zhang.)

Q. Zhang, Y. Yang, and E. Young are with the Faculty of Engineering and Environment, Institute of Cryogenics, University of Southampton, Southampton SO17 1BJ, U. K (e-mail: qz9g13@ soton.ac.uk; y.yang@ soton.ac.uk; E.A. Young@soton.ac.uk).

L. Cavalucci is with the Department of Electrical, Electronic, and Information Engineering, University of Bologna, Bologna 40136, Italy (e-mail: lorenzo.cavallucci3@unibo.it).

A. Kario and W. Goldacker are with the Department of Superconducting Materials and Energy Applications, Institute for Technical Physics, Karlsruhe Institute of Technology, Karlsruhe 76344, Germany (e-mail: anna.kario@kit.edu; wilfried.goldacker@kit.edu).

A. Usoskin is with the Bruker HTS GmbH, Alzenau 63755, Germany (e-mail: alexander.usoskin@bruker.com).

L. Bottura is with CERN, CH-1211 Geneva 23, Switzerland (e-mail: Luca.Bottura@cern.ch).

Color versions of one or more of the figures in this paper are available online at http://ieeexplore.ieee.org.

Digital Object Identifier 10.1109/TASC.2018.2799140
[9]-[11] becomes impractical for Roebel cables with long current sharing length [2].

In the present work, quench studies on Roebel cables were carried out on a simple pancake coil in order to incorporate a cable length for a full development of current sharing among strands. Although the simplicity of $1 \mathrm{~d}$ quench of adiabatic conductors is lost through lateral thermal contacts, the quench of a Roebel pancake coil is actually more relevant to real applications and the added complexity can be readily dealt with by suitably developed computer modelling.

We present in this paper the details of the preparation of the pancake coil, localized heater, and instrumentation setup of voltage taps and thermometers. Subsequently the preliminary experimental results on the cable performance and quench measurements at $77 \mathrm{~K}$ in liquid nitrogen are described.

\section{Methods And Experimental Details}

\section{A. Roebel Pancake Coil}

A piece of $2 \mathrm{~m}$ long Roebel cable (see Fig. 1) with 15 strands (5.5 mm wide) of punched 2G YBCO tapes (Bruker EST) was wound into a simple pancake coil of 7 turns. The cable of a transposition pitch of $226 \mathrm{~mm}$ was assembled at KIT. A length of $200 \mu \mathrm{m}$ thick fiberglass ribbon was co-wound as the electrical insulation layer. The inner diameter of the pancake coil was $72 \mathrm{~mm}$ to accommodate, for every strand on the inner turn, a section of direct soldered contact between the superconductor side and the copper former (see Fig. 2). To match the thermal contraction of the Roebel to the former, a G10 core was inserted inside the copper former. On the penultimate turn a copper ring mounted on a copper disk was slid over. The last turn on the outside was wound and soldered to the copper ring. This configuration allows direct current injection to the superconductor side of the Roebel strands. A stainless steel shim was wound around the last turn of the Roebel cable prior to soldering. Current injection was via two, half meter long, $16 \mathrm{~mm}$ diameter copper bars (see Fig. 2). The coil was vacuum impregnated with epoxy (StycastTM) [12].

\section{B. Instrumentation}

At the 4th turn of the coil, a miniature heater $(33 \Omega)$ was attached to strand 7 at its transposition on the middle inside face of the cable (see Fig. 1). A T-type thermocouple was soldered on the strand next to the heater. An epoxy impregnated fiberglass 


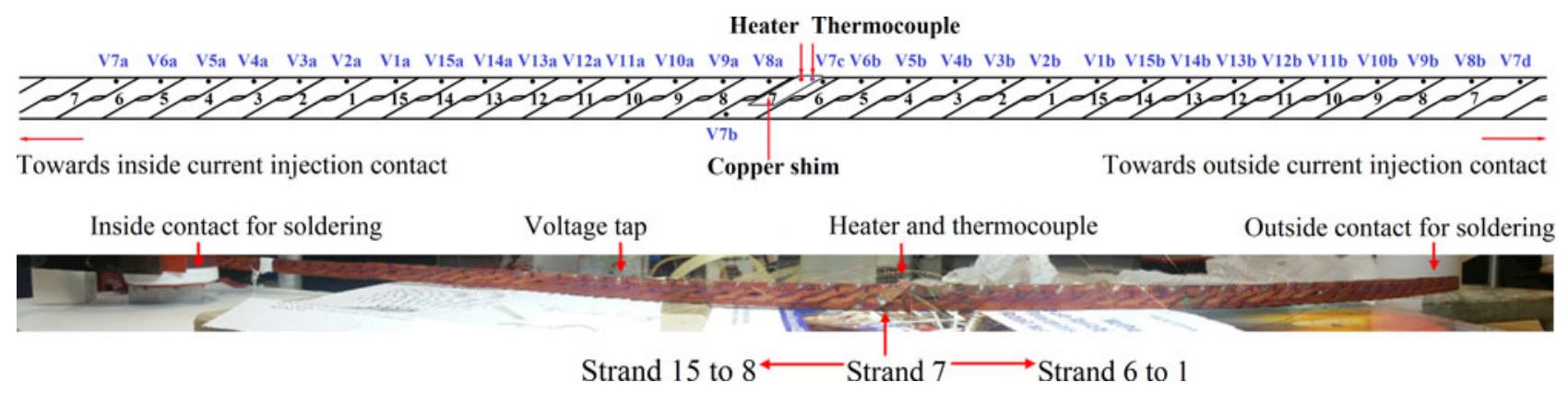

Fig. 1. The whole scale view of the Roebel cable instalment.

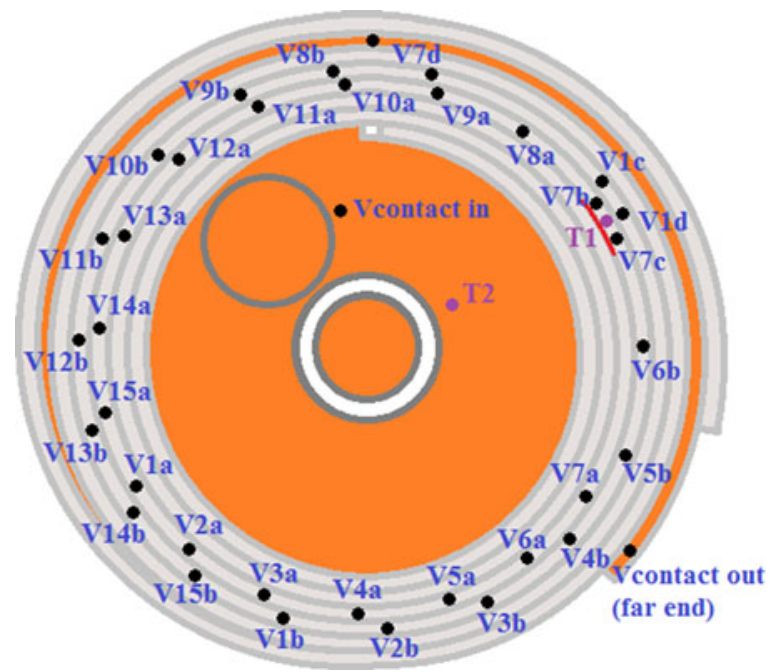

Fig. 2. Schematic drawing of the Roebel pancake coil to show the voltage taps (black points), heater (red line) and thermal couple (purple points).

thermal insulation layer $20 \mathrm{~mm}$ long, $6 \mathrm{~mm}$ wide and $2 \mathrm{~mm}$ thick was applied over the heater and thermocouple. A pair of voltage taps (V7b and V7c in Fig. 1) were soldered to the strand on either side of transposition section enclosing the heater. A further pair of taps (V7a and V7d) were soldered one pitch length away from V7b and V7c towards the inner and outer contacts.

For each of the remaining 14 strands within V7a and V7d, a voltage-tap pair (Vna and $\mathrm{Vnb}, \mathrm{n}=1 . .6,8 . .15$ ) separated by one pitch length was soldered on either side of the heater. The signal voltage-tap pair (Vna and $\mathrm{Vnb}, \mathrm{n}=1 . .6,8 . .15$ ) separated by pitch length was soldered on either side of the heater. The signal wires for each measured voltage pair $\mathrm{V} n(\mathrm{~V} n \mathrm{a}-\mathrm{V} n \mathrm{~b})$ were counter-wound along the respective cable path and the then twisted together to compensate the inductive voltage.

Using voltage taps Vcin and Vcout on the copper former and the end of copper ring respectively, the contact voltages for each strand were also measured with the corresponding voltage-tap pair (Vcin -Vna and Vcout -Vnb).

\section{Experimental}

The preliminary tests presented here were performed with the Roebel pancake coil submerged in liquid nitrogen at $77 \mathrm{~K}$.

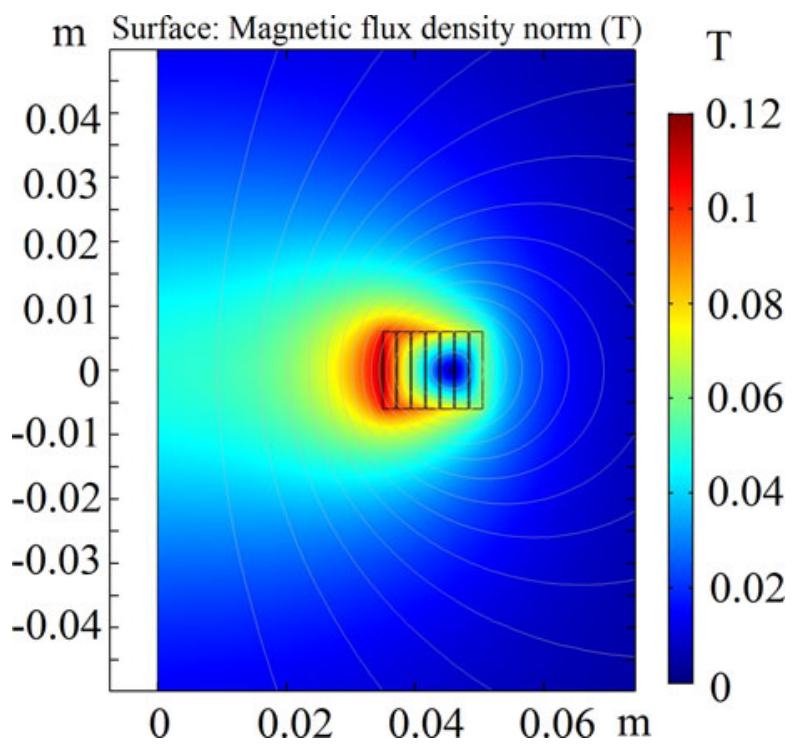

Fig. 3. Magnetic field density map and line of forces of the Robel contact pancake coil at $450 \mathrm{~A}$ (Left side: inside contact and right side: outside).

The voltage-tap pairs were used for the measurement of the critical current and $V I$ characteristics. The contact resistance was also measured simultaneously. Steady state local heating with the heater on strand 7 was used to extend the critical current measurement to higher temperatures for further corroboration.

Quench measurements were conducted at a given transport current by applying a heat pulse to the localized heater on strand 7 , which the heat pulse was trigged by applying a constant DC voltage $(4.5 \mathrm{~V})$. The minimum quench energy was determined by increasing the pulse power intensity and/or pulse duration until a thermal run-away.

\section{RESULTS AND DISCUSSION}

\section{A. Critical Current}

The original $2 \mathrm{G}$ YBCO tape has a homogeneous critical current of $130 \mathrm{~A}( \pm 10 \%)$ along the length at $77 \mathrm{~K}$ in self-field, corresponding to $I_{C} \sim 65 \mathrm{~A}$ for the punched strands [13]. The pancake coil field distribution at $450 \mathrm{~A}$ in Fig. 3 shows the maximum parallel and perpendicular fields are located at the inner most, $0.12 \mathrm{~T}$, and the edge of turns $3 / 4,0.08 \mathrm{~T}$ respectively. 


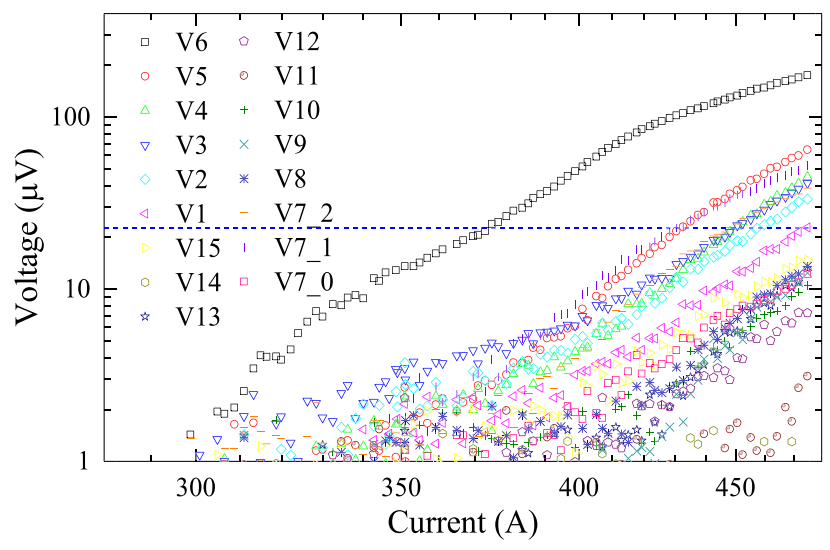

Fig. 4. Strand voltages as a function of current between $300 \mathrm{~A}$ to $470 \mathrm{~A}$.

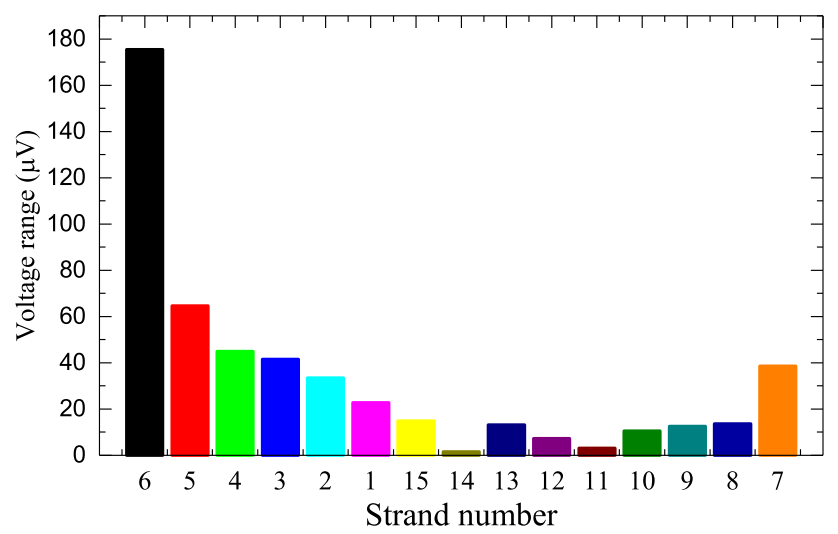

Fig. 5. Voltage across of each strand (2 pitches) across the same current ramping range from $300 \mathrm{~A}$ to $470 \mathrm{~A}$.

The critical current of short single strands was measured in liquid nitrogen at $77 \mathrm{~K}$. The results confirmed the single strand $I_{C}$ (self-field) $=63 \mathrm{~A}, I_{C}\left(B_{\|}=0.12 \mathrm{~T}\right)=30 \mathrm{~A}$ and $I_{C}\left(B_{\perp}=0.08 \mathrm{~T}\right)=33 \mathrm{~A}$. The critical current of the pancake coil is likely to be limited by the parallel field on the inner turn, which for 15 strands should be $450 \mathrm{~A}$.

The voltage-current $(V I)$ characteristics of all the strands on turns 2-4 are shown in Fig. 4, where non-linear increases of voltage with current can be seen on all strands starting at different currents. The nominal "critical current" by strand voltages at $1 \mu \mathrm{Vcm}^{-1}$ is about $370 \mathrm{~A}$ using $\mathrm{V} 6$ and $>470 \mathrm{~A}$ for strands $8-15$. Strand 6 showed the highest voltage with an onset current around only $300 \mathrm{~A}$ - a nominal $20 \mathrm{~A}$ per tape. The remaining strands exhibited different onset current ranging from $310 \mathrm{~A}$ to $450 \mathrm{~A}$. The voltage span of each strand between $300 \mathrm{~A}$ and $470 \mathrm{~A}$ are shown as a bar-chart in Fig. 5. The $\mathrm{x}$ axis is plotted in the sequence the strands transpose at the inner face in turn 2. The strand furthest towards the inside exhibit the highest voltages. The pitch length is the same as the inner solder joint length (turn 1). Consequently strand 6 transposes on the inside twice in turn 2. As a result, the voltage taps on strand 6 are closer to the inside. Such a voltage pattern is consistent with a higher parallel magnetic field on the inner turns which strand 6 experiences to a greater degree.

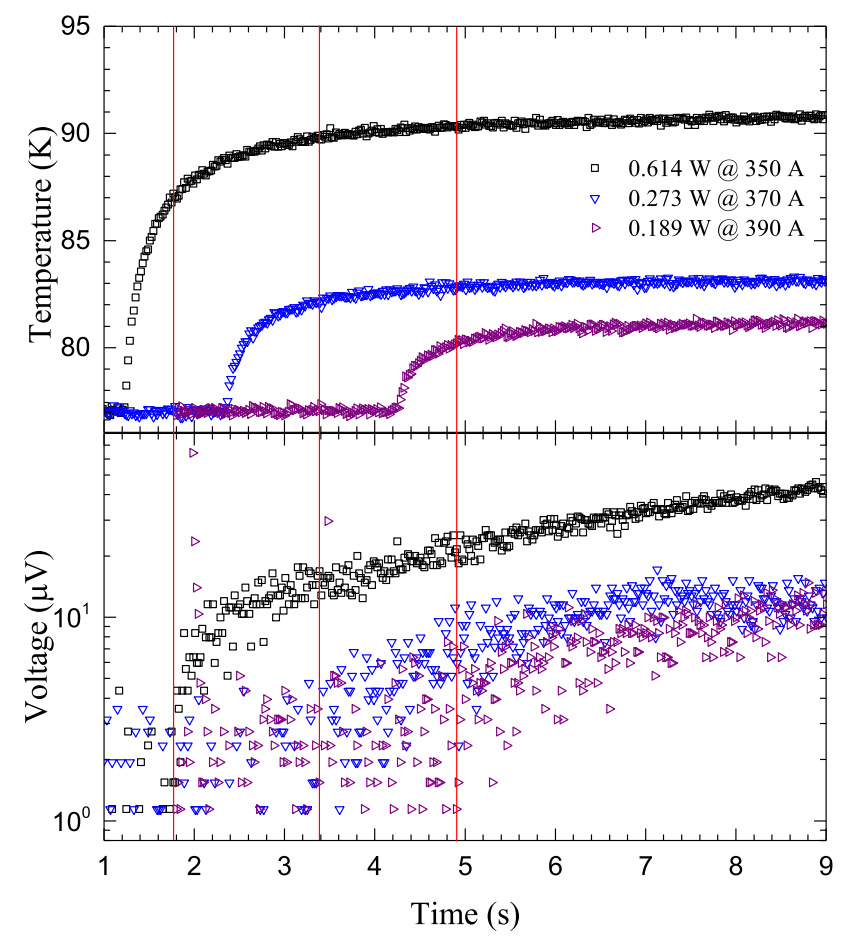

Fig. 6. Upper pane: temperature rise on strand 7 due to different heater power and lower: voltage V7_0 around the hot spot at the corresponding heating power and transport current.

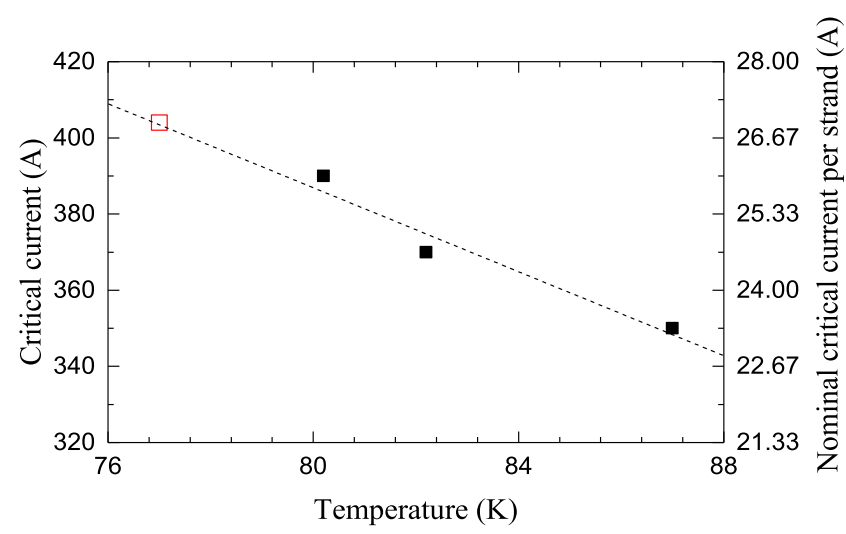

Fig. 7. Nominal critical current at different temperature.

The non-uniform critical current among the strands makes it difficult to determine the overall critical current of the coil. To further ascertain the nominal strand critical current, different power was applied to the heater on strand 7 at different transport current. The voltages onset for different temperatures and current in Fig. 6 leads to a local critical current as determined by V7_0 as a function of temperature shown in Fig. 7, where the right axis corresponds to the nominal critical current per strand, which is consistent with that at $77 \mathrm{~K}$, red open square from Fig. 4.

Despite the different voltages among the strands, it is important to note that the pancake coil was found to be stable at $440 \mathrm{~A}$ as shown in Fig. 8 where all the voltages are settled without signs of thermal runaway. 


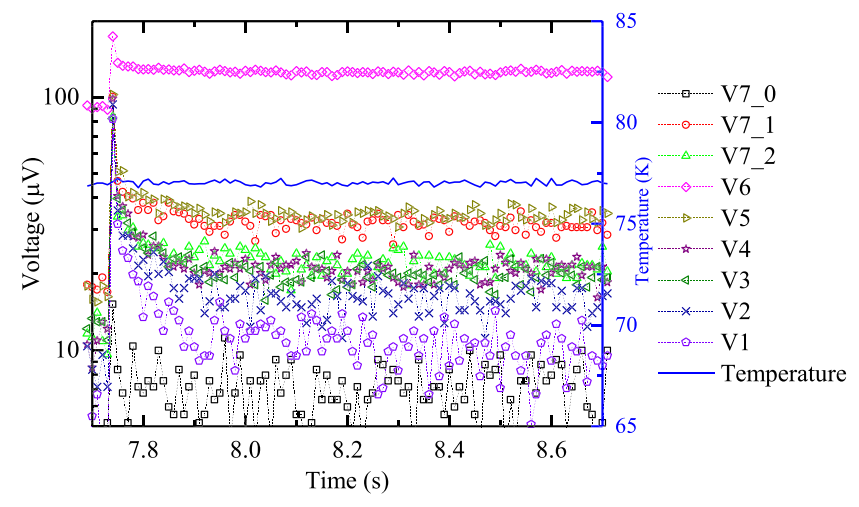

Fig. 8. All strand voltages at $440 \mathrm{~A}$ (Strand 8-15 voltages are below $10 \mu \mathrm{V}$ ).

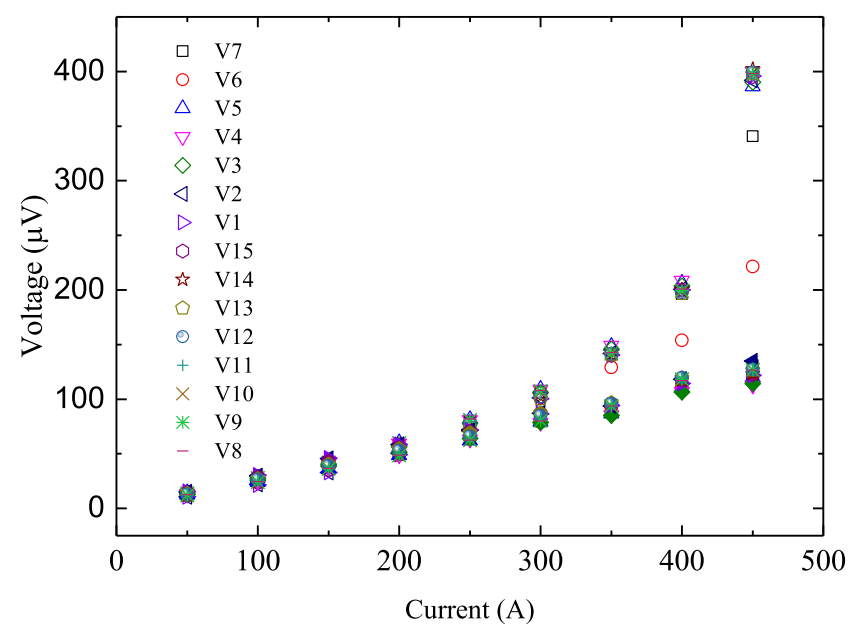

Fig. 9. Inner (open symbols) and outer (filled symbols) contact voltages of all the strands as a function of current.

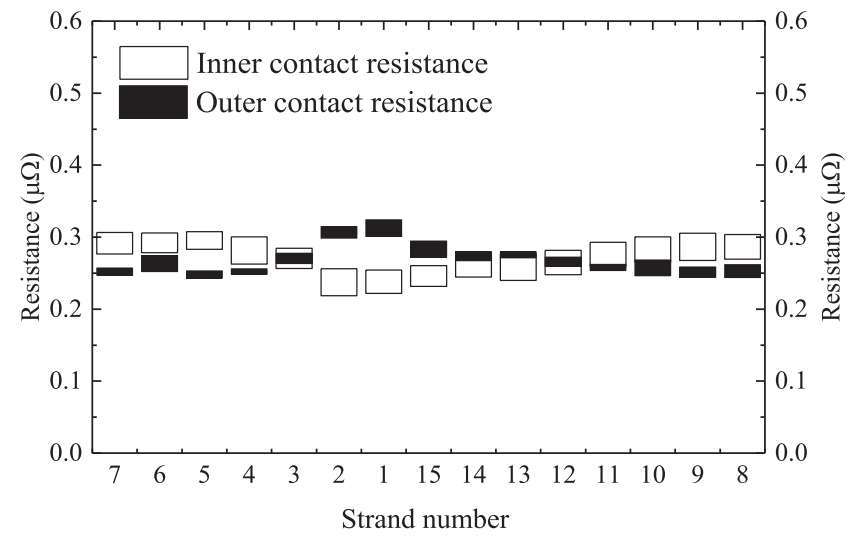

Fig. 10. Apparent inner (open bars) and outer (filled bars) contact resistances according to voltage taps on different strands.

\section{B. Contact Resistance}

Both inside and outside contact voltages of each strand were measured as a function of current and shown in Fig. 9. The inner contacts (open bars in Fig. 10) appeared to have a minimum mirroring the strand voltages on the 3rd turn shown in Fig. 5, while a matching maximum was found in the outer contacts. The minimum in the inner contacts is likely a reflection of the

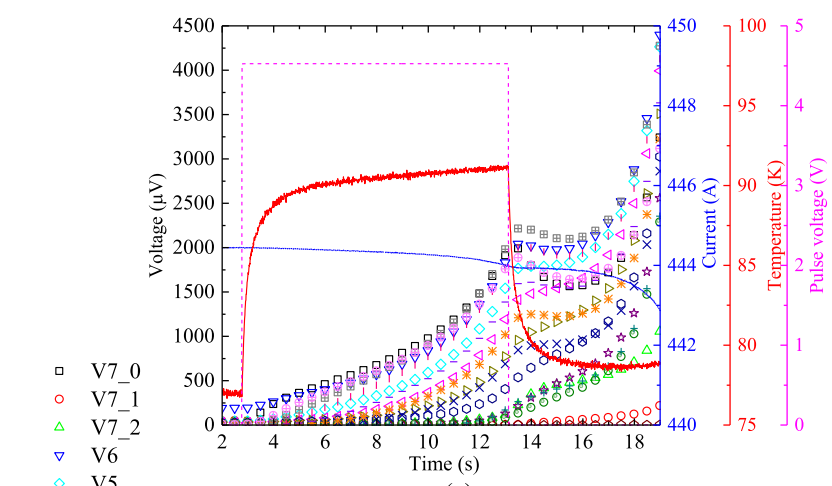

(a)

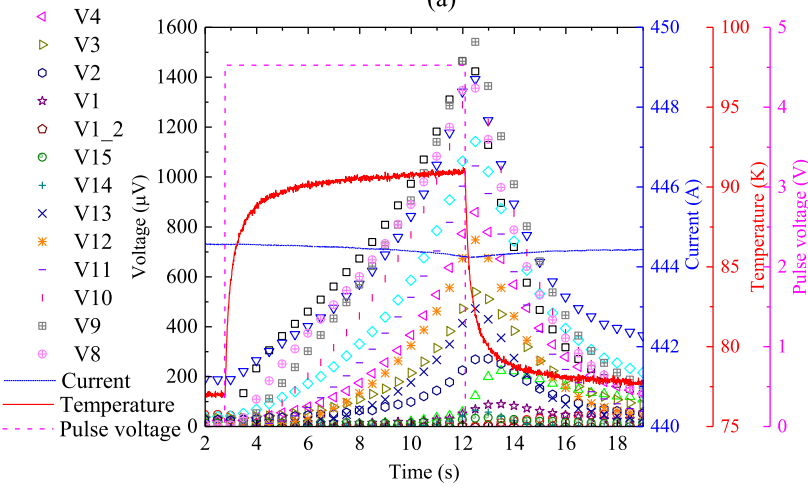

(b)

Fig. 11. The tracing of voltage of each strand, current, temperature and pulse voltage against time for (a). coil quench and (b). coil recover after heating pulse cutting off.

field effect on the critical current at the inner turns. The low voltage strands might have a larger current share which leads to the maximum shown on corresponding outer contacts. The overall inner or outer contact resistance is $0.2-0.3 \mu \Omega$.

It's worth noting the nonlinearity with current shown by the inner contact voltages above $200 \mathrm{~A}$ in Fig. 9. The inner contact voltage of strand 6 increases less with increasing total current compared with other strands. When the total DC current is below 200 A, the current in each inner strand contact should only depend on the contact resistance. When the total current is above $200 \mathrm{~A}$, the current in each inner strand contact is mainly driven by the critical current of the inner turn, where the parallel field is the highest. Consequently despite the higher strand 6 voltage above 200 A the relatively lower contact voltage means it is carrying less current and is being stabilized by current sharing in the other strands.

\section{Quench Measurement}

Although high stability is expected for the Roebel pancake coil in liquid nitrogen, it was found that quench could still be triggered by point-like disturbances with localized heat deposition. A trial of quench measurements for this Robel pancake coil was undertaken in LN2 with a transport current around 450 A with several heat pulses $(4.5 \mathrm{~V})$. The strand voltage, transport current, temperature and pulse voltage of two examples are traced and shown in Fig. 11. The first trial shown in Fig. 11(a) was conducted with an $11.3 \mathrm{~s}$ heat pulse with $7 \mathrm{~J}$. The 
temperature (red line) increases to above $90 \mathrm{~K}$. All the strands quench shown by all voltages continue to increase after the heat is off; and the current (blue dot line) drops below $443 \mathrm{~A}$, which implies a continued current sharing with the shunt load. The second trial of quench measurement shown in Fig. 11(b) was conducted with a $9.7 \mathrm{~s}$ heat pulse of $6 \mathrm{~J}$. The trend of voltage traces, temperature and current coincide with the first example during the period of heat applied. After the heat pulse is off, the strand voltage, current and temperature recover.

\section{CONCLUSION}

The performance and quench characteristics of this pancake coil is presented in this paper. The critical current of this Roebel pancake coil is around $370 \mathrm{~A}$ with a higher parallel magnetic field effect on the inner turns. The overall inner and outer contact resistances are similar at $0.2-0.3 \mu \Omega$. All the strands have a thermal run away when the heat energy is above $7 \mathrm{~J}$. In future, more quench measurement will be undertaken with a boundary condition close to adiabatic with different fractions of the critical current in a $10 \mathrm{~T}$ variable temperature insert rig.

\section{REFERENCES}

[1] G. A. Kirby et al., "First cold powering test of REBCO roebel wound coil for the EuCARD2 future magnet development project," IEEE Trans. Appl. Supercond., vol. 27, no. 4, Jun. 2017, Art. no. 4003307.

[2] W. Goldacker et al., "Status of high transport current ROEBEL assembled coated conductor cables," Supercond. Sci. Technol., vol. 22, no. 3, 2009, Art. no. 034003.

[3] G. A. Kirby et al., "Accelerator-quality HTS dipole magnet demonstrator designs for the EuCARD-2 5-T 40-mm clear aperture magnet," IEEE Trans. Appl. Supercond., vol. 25, no. 3, Jun. 2015, Art. no. 4000805.
[4] J. Fleiter, A. Ballarino, L. Bottura, W. Goldacker, and A. Kario, "Characterization of roebel cables for potential use in high-field magnets," IEEE Trans. Appl. Supercond., vol. 25, no. 3, Jun. 2015, Art. no. 4802404.

[5] W. Goldacker et al., "High current DyBCO-ROEBEL assembled coated conductor (RACC)," J. Phys. Conf. Ser., vol. 43, no. 1, pp. 901-904, 2006.

[6] S. Terzieva et al., "Transport and magnetization AC losses of ROEBEL assembled coated conductor cables: Measurements and calculations," $\mathrm{Su}$ percond. Sci. Technol., vol. 23, no. 1, 2010, Art. no. 014023.

[7] N. Glasson, M. Staines, R. Buckley, M. Pannu, and S. Kalsi, "Development of a 1 MVA 3-phase superconducting transformer using YBCO Roebel cable," IEEE Trans. Appl. Supercond., vol. 21, no. 3, pp. 1393-1396, Jun. 2011.

[8] N. Amemiya, T. Tsukamoto, M. Nii, T. Komeda, T. Nakamura, and Z. Jiang, "Alternating current loss characteristics of a Roebel cable consisting of coated conductors and a three-dimensional structure," Supercond. Sci. Technol., vol. 27, no. 3, 2014.

[9] E. A. Young, S. Chappell, I. Falorio, and Y. Yang, "Quench characteristics of a Cu-stabilized 2G HTS conductor," IEEE Trans. Appl. Supercond., vol. 21, no. 3, pp. 3062-3065, Jun. 2011.

[10] I. Falorio, E. A. Young, and Y. Yang, "Quench characteristic and minimum quench energy of 2G YBCO tapes," IEEE Trans. Appl. Supercond., vol. 25 , no. 3, Jun. 2015, Art. no. 6605505.

[11] E. A. Young, S. Chappell, I. Falorio, and Y. Yang, "Quench characteristics of a stabilizer-Free 2G HTS conductor," IEEE Trans. Appl. Supercond., vol. 19, no. 3, pp. 2500-2503, Jun. 2009.

[12] W. Bailey, H. Wen, M. Al-Mosawi, K. Goddard, and Y. Yang, "Testing of a lightweight coreless HTS synchronous generator cooled by subcooled liquid nitrogen," IEEE Trans. Appl. Supercond., vol. 21, no. 3, pp. 11591162, Jun. 2011

[13] A. Kario, "HTS Roebel cables for the EuCARD-2 'future magnets'," in Proc. 3rd EuCARD-2 Annu. Meet., p. 5, 2016. [Online]. Available: https://indico.cern.ch/event/489475/contributions/1166833/attachments/ 1263291/1868932/23.04.2016_Malta_Kario.pdf. Accessed on: Dec. 13, 2017. 